J. Lake Sci. (湖泊科学) , 2012, 24(6): 843-848

http: //www. jlakes.org. E-mail : jlakes@niglas.ac.cn

(c) 2012 by Journal of Lake Sciences

\title{
添加固体碳源对垂直流人工湿地污水处理效果的影响"
}

\author{
肖 蕾 ${ }^{1,2}$, 贺 锋 ${ }^{1 * *}$, 梁 雪 $^{1,2}$, 黄福青 ${ }^{1,2}$, 徐 栋 $^{1}$, 吴振斌 ${ }^{1}$ \\ (1:中国科学院水生生物研究所淡水生态和生物技术国家重点实验室,武汉 430072) \\ (2: 中国科学院研究生院,北京 100049)
}

\begin{abstract}
摘 要: 以垂直流人工湿地小试系统为研究对象, 探讨了不同位置 (表层、上层、中层和下层) 添加固体碳源对系统氮、磷 及 $\mathrm{COD}_{\mathrm{Cr}}$ 去除效果的影响. 结果表明: 湿地下层硝态氮去除率低于中层, 最适宜碳源添加位置为垂直流人工湿地中、下层. 添加碳源系统中, 碳源添加位置为表层的系统 $\mathrm{COD}_{\mathrm{Cr}}$ 去除率最高, 各系统出水 $\mathrm{COD}_{\mathrm{Cr}}$ 浓度均低于进水, 不引起系统出水中 $\mathrm{COD}_{\mathrm{Cr}}$ 浓度的增加. 添加碳源显著提高脱氮除磷效果, 碳源添加位置为下层的系统 TN 去除率最高, 碳源添加位置为表层 的系统氨氧化作用明显, 出水铵态氮浓度最高, 各系统对亚硝态氮和硝态氮去除率差异不明显, 但对硝态氮都表现出良 好的去除效果. 碳源添加位置为下层的系统硝化作用最完全, TP 去除能力也显著优于其他各系统. 添加碳源至垂直流人 工湿地下层可以达到同步脱氮除磷的效果.
\end{abstract}

关键词：固体碳源;氮; 反硝化;垂直流人工湿地;污水处理

\section{Effect of the solid carbon sources addition on performance of wastewater treatment effi- ciency in vertical flow constructed wetland}

\author{
XIAO Lei ${ }^{1,2}$, HE Feng ${ }^{1}$, LIANG Xue ${ }^{1,2}$, HUANG Fuqing ${ }^{1,2}$, XU Dong ${ }^{1} \&$ WU Zhenbin ${ }^{1}$ \\ (1: State Key Laboratory of Freshwater Ecology and Biotechnology, Institute of Hydrobiology, Chinese Academy of Sciences, \\ Wuhan 430072 , P. R. China) \\ (2: Graduate University of Chinese Academy of Sciences, Beijing 100049, P. R. China)
}

\begin{abstract}
The optimum of carbon sources adding location and the effects of adding to different locations (surface, the upper, middle and lower layers) on the removal efficiency of nitrogen, phosphorus and $\mathrm{COD}_{\mathrm{Cr}}$ were investigated in small-scale vertical flow constructed wetlands. The results showed that the removal efficiency of the nitrate nitrogen in the lower layer was lower than that in the middle layer, and the most suitable location for carbon sources supplement was the middle and lower layer of the vertical flow constructed wetlands. Among the systems with carbon sources added, the one which added the carbon sources to the surface layer exhibited the highest removal efficiency of $\mathrm{COD}_{\mathrm{Cr}}$, and the concentration of $\mathrm{COD}_{\mathrm{Cr}}$ of the effluent of each system were lower than the influent, not causing the concentration of $\mathrm{COD}_{\mathrm{Cr}}$ increasing of the effluent of the systems. Adding carbon sources can significantly improve the removal efficiency of nitrogen and phosphorus. The system which added the carbon sources to the lower layer showed the highest removal efficiency of total nitrogen. The system which added the carbon sources to the surface layer showed significant ammoxidation, and the concentration of ammonia nitrogen of the effluent was highest. The removal efficiency of each system showed no significant difference in the removal efficiency of nitrite and nitrate nitrogen, and all showed great removal efficiency of nitrate nitrogen. The system which added the carbon sources to the lower layer showed the most complete nitrification, and the removal efficiency of total phosphorus was also significantly better than that of other systems. Adding carbon sources to the lower layer of the vertical flow constructed wetland can achieve the effect of simultaneous removal of nitrogen and phosphorus.
\end{abstract}

Keywords: Solid carbon sources; nitrogen; denitrification; vertical flow constructed wetland; wastewater treatment

* 国家自然科学基金项目 $(51178452,50808172)$ 、湖北省杰出青年基金项目(2010CDA093)、国家科技支撑计划项目 (2012BAJ21B03-04) 和国家水体污染控制与治理科技重大专项项目 (2012ZX07101007-005,2009ZX07106-002) 联 合资助. 2012-01-05 收稿;2012-04-12 收修改稿. 肖蕾,女,1985 年生,硕士研究生; E-mail : xiaolei-e617@ 163. com.

** 通信作者;E-mail:hefeng@ ihb. ac. cn. 
人工湿地是 $1970 \mathrm{~s}$ 发展起来的一种污水处理工艺, 它利用基质、微生物和植物组成的复合生态系统的物 理、化学和生物作用实现对污水的净化 ${ }^{[1]}$. 因其具有投资省、管理简便、运行费用低等优点成为发展极为迅 速的污水处理技术, 同时在水体修复与生态建设方面也发挥着重要作用 ${ }^{[2]}$. 人工湿地可以有效地去除污染 水体中的有机物 ${ }^{[3-10]}$, 具有较好脱氮潜能, 但是在实际运行的人工湿地的相关报道中, 不同类型人工湿地对 氮的去除效果差异很大 ${ }^{[11]}$, 人工湿地脱氮效果的差异与水力条件、运行温度、溶解氧等因素有关. 研究表 明 ${ }^{[12-13]}$, 在以脱氮为主要目的的人工湿地中, 碳源是湿地脱氮的主要限制性因素, 而通过投加外源性碳源可 以显著提高湿地的脱氮效果. 目前, 国内外学者针对人工湿地反硝化碳源不足的问题, 多采用向进水中投加 葡萄糖、甲醇、醋酸钠等液态有机碳的方法 ${ }^{[14-15]}$. 但液态有机碳反应速度快, 导致需要经常补充碳源, 因此开 展纤维素等固体碳源的添加研究是一种有益的尝试 ${ }^{[16]}$, 已有研究发现, 向人工湿地中添加水生植物材料或 其残体可提高系统反硝化脱氮效能 ${ }^{[17]}$. 但植物材料作为有机碳源补充于垂直流态的人工湿地中的相关研究 较少, 在碳源添加量, 添加方式等方面缺乏系统的报道. 本实验探讨了在垂直流人工湿地不同位置添加千屈 菜作为外加碳源时, 湿地系统中有机物及 $N 、 P$ 的去除效果及机理, 以期为提高人工湿地脱氮除磷效果提供 理论依据.

\section{1 材料与方法}

\section{1 实验装置}

垂直流人工湿地系统共 5 套, 采用内径 $250 \mathrm{~mm}$ 的 PVC 管制成, 其中管高 $750 \mathrm{~mm}$, 填料高度为 $600 \mathrm{~mm}$, 填料为粒径 $5 \mathrm{~mm}$ 的生物陶粒, 取自河南巩义. 其中底部 $150 \mathrm{~mm}$ 厚的填料作为排水层, 管顶留有 $100 \mathrm{~mm}$ 的 布水区, 另有 $50 \mathrm{~mm}$ 的超高. 自管顶向下每隔 $150 \mathrm{~mm}$ 设置 1 个分层水样采集口、 1 个碳源添加装置及 1 个测 压管接口, 系统中种植美人蕉 (Canna generalis). $1^{\#}$ 系统为对照组, 不添加碳源材料, $2^{\#} \sim 5^{\#}$ 系统添加碳源位 置分别为 $a 、 b 、 c 、 d$ (距填料表面深度分别为 $0 、 15 、 30 、 45 \mathrm{~cm}$, 即表层、上层、中层、下层, 图 1).

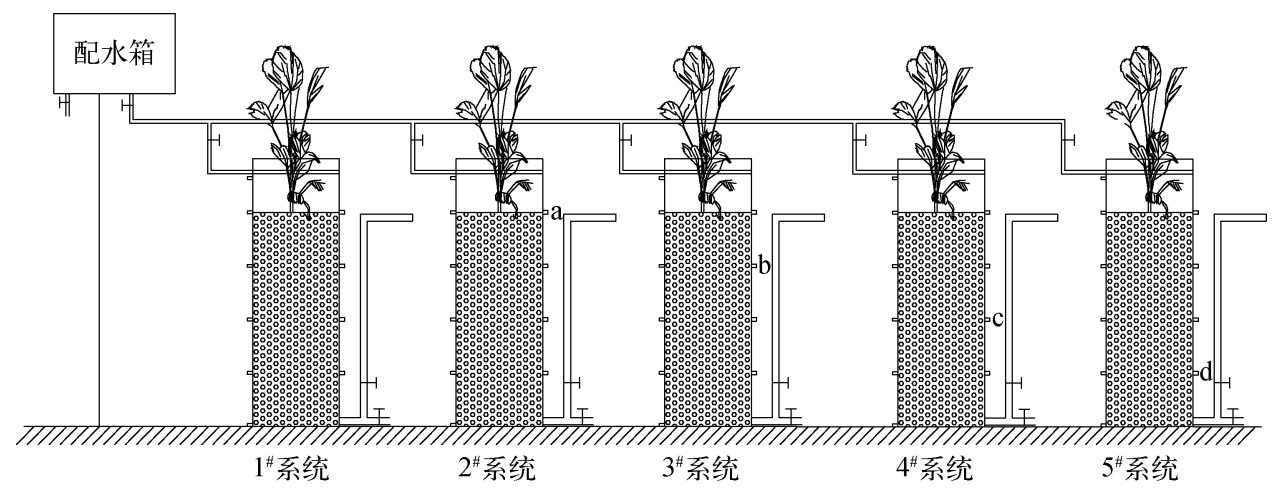

图 1 垂直流人工湿地试验装置示意图

Fig. 1 Schematic diagram of the experimental model of vertical flow constructed wetland

\section{2 实验材料}

本实验选取的反硝化植物碳源材料千屈菜 (Lythrum salicaria) 采自中国科学院水生生物研究所官桥实 验基地, 在实验室内将其切成 $2 \mathrm{~cm} \times 2 \mathrm{~cm}$ 的片状, 置于 $1 \% \mathrm{NaOH}$ 溶液中浸泡 $24 \mathrm{~h}$, 用自来水洗净后在 $78^{\circ} \mathrm{C}$ 烘箱中烘 $72 \mathrm{~h}$, 放在烘箱内冷却, 然后装人样品袋置于干燥器中备用.

\section{3 系统运行条件}

系统进水采用自配污水, 其中碳源主要采用蛋白胨、牛肉膏, 氮源主要采用氯化铵, 磷源主要采用磷酸二氢 钾, 微量元素主要采用无水氯化钙和无水硫酸镁. 实际配制后的污水化学需氧量 $\left(\mathrm{COD}_{\mathrm{Cr}}\right)$ 浓度为 $112.33 \pm$ $50.28 \mathrm{mg} / \mathrm{L}$, 总氮 $(\mathrm{TN})$ 浓度为 $36.94 \pm 3.12 \mathrm{mg} / \mathrm{L}$, 总磷 $(\mathrm{TP})$ 浓度为 $4.02 \pm 0.75 \mathrm{mg} / \mathrm{L}$, 铵态氮 $\left(\mathrm{NH}_{4}^{+}-\mathrm{N}\right)$ 浓 度为 $30.58 \pm 4.51 \mathrm{mg} / \mathrm{L}$; 水温为 $18.10 \pm 0.64^{\circ} \mathrm{C} .1^{\#}$ 系统不添加碳源, $2^{\#} \sim 5^{\#}$ 系统添加碳源位置分别为表层、 
上层、中层和下层. 碳源添加量为 $160 \mathrm{~g}$, 均匀布于填料横断面. 系统采用间歇式进水, 水力负荷为 $300 \mathrm{~mm} / \mathrm{d}$. 每两天定时进水 1 次, 水力停留时间 (HRT) 设定为 $48 \mathrm{~h}$, 日进水量为 $15 \mathrm{~L}$. 系统建成后, 稳定 2 月后开始本实 验研究.

\section{4 主要监测指标及方法}

$1^{\#}$ 系统在进水采样口及各分层水样采集口进行采样, $2^{\#} \sim 5^{\#}$ 系统在进水采样口及每套系统出水口进行 水样的采集, 采样频率为每两天 1 次. 温度、pH 值、溶解氧 (DO) 等采用 Thermo Orion Five-Star 型便携式测定 仪测定. $\mathrm{COD}_{\mathrm{Cr}}$ 采用哈希密封消解比色法 ( DR/2010, HACH, USA); TN 采用过硫酸钾紫外分光光度法; TP 采 用过硫酸钾钿锑抗分光光度法; $\mathrm{NH}_{4}^{+}-\mathrm{N}$ 采用纳氏试剂分光光度法; 硝态氮 $\left(\mathrm{NO}_{3}^{-}-\mathrm{N}\right)$ 采用紫外分光光度法; 亚硝态氮 $\left(\mathrm{NO}_{2}^{-}-\mathrm{N}\right)$ 采用 $\mathrm{N}-(1$-荎基 $)$-乙二胺光度法 ${ }^{[18]}$.

\section{5 数据处理}

各阶段实验数据采用 SPSS 13.0 统计软件进行 One-way ANOVA 方差分析, 用 LSD 法进行多个样本的均 数比较, 显著性水平设为 0.05 .

\section{2 结果与分析}

\section{1 适宜碳源添加位置探寻}

本实验结果基于 $1^{\#}$ 系统的运行效果. 垂直流人工湿地硝态氮去除率的沿程变化可以看出, 上层、中层和 下层硝态氮的去除均取得了较好的效果, 去除率分别达到 $87.39 \%$ 、 $89.67 \%$ 和 $88.04 \%$, 出水硝态氮浓度均 降至 $0.5 \mathrm{mg} / \mathrm{L}$ 以下, 而表层的硝态氮去除率仅为 $4.56 \%$ (图 2). 贺锋等 ${ }^{[6]}$ 在复合垂直流人工湿地基质内反 硝化作用的空间变异研究中发现, 反硝化作用强度在基质各层都相差不大, 在下行流池 $0 \sim 10 \mathrm{~cm}$ 层最低, 这 与本实验结果一致. 表层硝态氮去除率与上层、中层、下层有显著性差异 $(P<0.05)$, 而上层、中层和下层之 间差异不显著 $(P>0.05)$.

沿着水流方向 $\mathrm{COD}_{\mathrm{Cr}}$ 浓度逐级降低, 进水 $\mathrm{COD}_{\mathrm{Cr}}$ 浓度为 $177 \mathrm{mg} / \mathrm{L}$, 表层、上层、中层和下层的 $\mathrm{COD}_{\mathrm{Cr}}$ 浓度 分别为 $163 、 62 、 54$ 和 $34 \mathrm{mg} / \mathrm{L}$ (图 2). 随着 $\mathrm{COD}_{\mathrm{Cr}}$ 浓度的降低, 下层的硝态氮去除率较中层降低了 $1.63 \%$, 这 可能是由于 $\mathrm{COD}_{\mathrm{Cr}}$ 浓度的降低导致微生物可利用的碳源缺乏, 从而阻碍了反硝化作用的进行, 因此认为碳源 的添加位置宜选择在垂直流人工湿地中、下层. 这与余丽华等 ${ }^{\left[{ }^{[19]}\right.}$ 的报道结果一致.

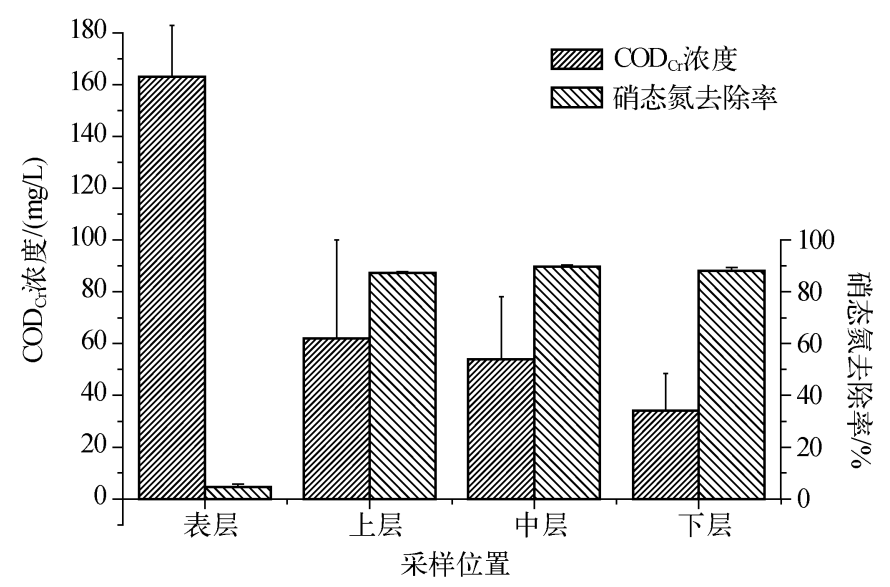

图 2 垂直流人工湿地 $\mathrm{COD}_{\mathrm{Cr}}$ 浓度及硝态氮去除率的沿程变化

Fig. 2 Variations of $\mathrm{COD}_{\mathrm{Cr}}$ concentrations and the removal rates of nitrate nitrogen along the distance in vertical flow constructed wetland

\section{2 碳源添加位置对 $\mathrm{COD}_{\mathrm{Cr}}$ 去除效果的影响}

系统进水 $\mathrm{COD}_{\mathrm{Cr}_{\mathrm{r}}}$ 浓度为 $98 \sim 188 \mathrm{mg} / \mathrm{L}, 1^{\#}$ 系统出水 $\mathrm{COD}_{\mathrm{Cr}}$ 浓度为 $8 \sim 96 \mathrm{mg} / \mathrm{L}$. 系统运行稳定后, $2^{\#} \sim 4^{\#}$ 系 
统 $\mathrm{COD}_{\mathrm{Cr}}$ 出水浓度均低于进水, 说明千屈菜植物材料是人工湿地系统可供选择的有机碳源材料, 添加后并没 有引起出水 $\mathrm{COD}_{\mathrm{Cr}}$ 浓度的增加 (图 3). 其中 $3^{\#}$ 和 $4^{\#}$ 系统, 在运行初期 $\mathrm{COD}_{\mathrm{Cr}}$ 出水浓度高于进水, 这可能是由 于运行初期植物材料有机碳释放过快而造成的. 各系统 $\mathrm{COD}_{\mathrm{Cr}}$ 出水浓度变化规律相似, 均表现为系统运行初 期 $\mathrm{COD}_{\mathrm{Cr}}$ 浓度急剧下降, 稳定一段时间后再下降, 系统运行初期 $\mathrm{COD}_{\mathrm{Cr}}$ 浓度迅速下降可能是由于气温较高的 原因, 稳定后继续降低可能是因为湿地系统中微生物群落结构逐渐稳定. 添加碳源的各系统与对照组之间 差异显著 $(P<0.05), 2^{\#} \sim 5^{\#}$ 系统间差异不显著 $(P=0.058)$.
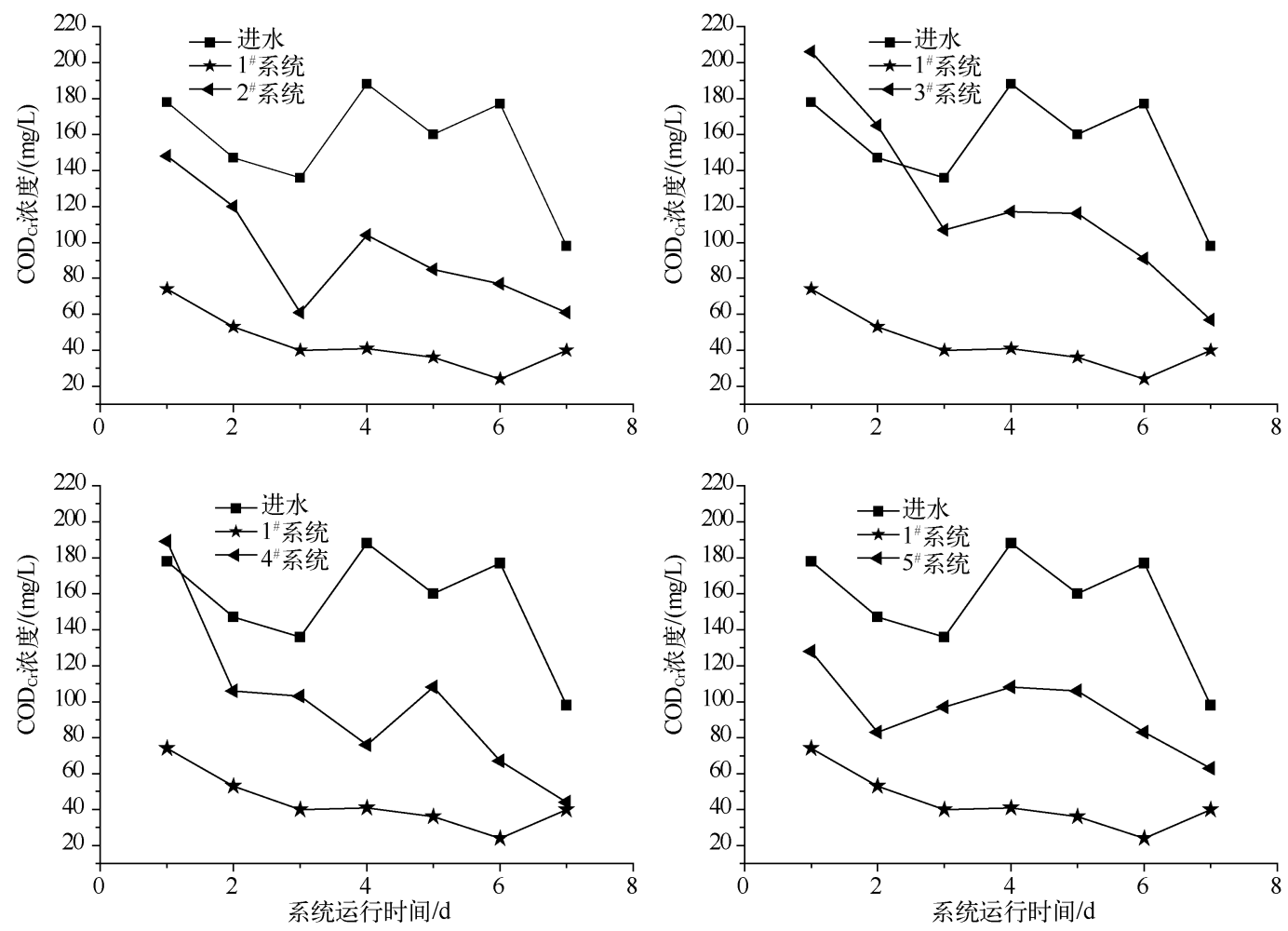

图 3 各系统出水 $\mathrm{COD}_{\mathrm{Cr}}$ 浓度变化

Fig. 3 Concentrations of $\mathrm{COD}_{\mathrm{Cr}}$ of the effluent of each system

$1^{\#} \sim 5^{\#}$ 系统出水 $\mathrm{COD}_{\mathrm{Cr}}$ 去除率分别为 $67.21 \% 、 36.56 \% 、 19.10 \% 、 28.29 \% 、 29.25 \% .1^{\#} \sim 3^{\#}$ 系统 $\mathrm{COD}_{\mathrm{Cr}}$ 去除率逐渐降低, $3^{\#} \sim 5^{\#}$ 系统之间升高, 这表明在碳源添加系统中, $2^{\#}$ 系统 $\mathrm{COD}_{\mathrm{Cr}}$ 去除率最高, $3^{*}$ 系统最低. 随 着水流方向, 硝化作用强度逐渐减弱, 反硝化作用略微增强, 湿地表层硝化作用明显, 消耗大量有机碳, 因此 $2^{\#}$ 系统表现为较高的 $\mathrm{COD}_{\mathrm{Cr}}$ 去除率; 湿地上层硝化作用迅速减弱, 反硝化作用增强不明显, 因此 $3^{\#}$ 系统 $\mathrm{COD}_{\mathrm{Cr}}$ 去除率低; 湿地中、下层硝化作用缓慢减弱, 反硝化作用增强, 因此 $4^{\#}$ 和 $5^{\#}$ 系统 $\mathrm{COD}_{\mathrm{Cr}}$ 去除率略有 提高.

\section{3 碳源添加位置对氮去除效果的影响}

硝化和反硝化作用是人工湿地除氮的主要途径, 污水进人湿地后先通过氨氧化作用将有机氮转化为铵 态氮, 然后通过硝化作用将铵态氮转化为亚硝态氮和硝态氮, 再经过反硝化作用将氮素转化成气态形式从 湿地中去除. $1^{\#} \sim 5^{\#}$ 系统出水 TN 浓度依次降低, 说明添加碳源后提高了湿地系统的脱氮效果 (图 4). 一般认 为, 氮从水中最终的去除主要依靠微生物的反硝化作用转化为气体逸出系统, 有机碳源是反硝化作用主要 的电子供体, 碳源的不足是制约反硝化的关键因素. 本实验中进水 $\mathrm{COD}_{\mathrm{Cr}} / \mathrm{TN}$ 仅为 3.03 , 是典型的低碳高氮 污水, 补充千屈菜植物材料为反硝化作用提供了充足的电子供体, 从而提高了系统的脱氮效果. 研究结果表 
明, 碳源添加位置为湿地下层时 TN 去除率 最高, 这与碳源添加位置选择实验结果吻 合. $5^{\#}$ 系统 TN 去除率分别比 $1^{\#} \sim 4^{\#}$ 系统提 高了 $3.80 \% 、 2.44 \% 、 1.40 \%$ 和 $0.87 \%$.

实验结果显示 5 套系统出水铵态氮浓 度均高于进水, 说明系统中氨氧化作用明 显. $2^{\#} \sim 5^{\#}$ 系统铵态氮出水浓度依次升高, 但都低于 $1^{\#}$ 系统, 这可能是由于湿地进水中 有机氮含量较高, 氨氧化作用产生大量铵态 氮. 同时添加碳源可以提高系统的反硝化作 用, 硝态氮及亚硝态氮的急剧减少促进硝化 作用的进行. 沿着水流方向硝化作用逐渐减 弱,因而 $2^{\#}$ 系统硝化作用最强, $5^{*}$ 系统硝化 作用最弱, $2^{\#} \sim 5^{\#}$ 系统的铵态氮浓度分别比 $1^{\#}$ 系统降低了 $2.13 、 1.50 、 1.20$ 和 $0.53 \mathrm{mg} / \mathrm{L}$ (图4).

各系统出水亚硝态氮浓度比进水高, 除 $2^{\#}$ 系统出水浓度与对照组相同外, $3^{\#} \sim 5^{\#}$ 系统出水浓度分别比对 照组降低 $0.03 、 0.02$ 和 $0.03 \mathrm{mg} / \mathrm{L}$, 说明添加碳源不会引起亚硝态氮的积累, 各系统出水浓度较为接近, 差 异不显著 $(P>0.05)$. 各系统对硝态氮表现出良好的去除效果, $1^{\#} \sim 5^{\#}$ 系统出水硝态氮去除率分别为 $88.72 \% 、 86.25 \% 、 85.78 \% 、 86.33 \%$ 和 $85.71 \%$,各系统间无显著差异 $(P>0.05)$.

依据各系统出水铵态氮占 $\mathrm{TN}$ 百分比含量, $2^{*} \sim 5^{*}$ 系统出水中 $\mathrm{TN}$ 中铵态氮所占比例依次升高, 分别为 $93.80 \% 、 96.78 \% 、 98.28 \%$ 和 $99.69 \%$, 可见 $5^{\#}$ 系统反硝化作用最完全, $4^{\#}$ 系统次之, 这与碳源添加位置选择 实验结果一致.

\section{4 碳源添加位置对 TP 去除效果的影响}

人工湿地对磷的去除主要通过基质的吸附、微生物以及植物的吸收作用. 研究认为 ${ }^{[20]}$, 随着有机物浓度 的增加, 基质对磷的吸附量降低, 而微生物的作用越来越明显. 磷能够取得良好去除效果的必要条件是聚磷 菌释磷必须充分, 而要实现充分释磷则需向聚磷菌提高充足的有机碳源 ${ }^{[21]} .1^{\#} \sim 5^{\#}$ 系统 TP 的去除率分别为 $39.71 \% 、 46.88 \% 、 51.87 \% 、 48.44 \% 、 60.41 \%$. 添加碳源后 TP 去除率得到显著提高 $(P<0.05), 2^{\#} \sim 4^{\#}$ 系统 对 TP 的去除没有显著差异 $(P>0.05), 5^{\#}$ 系统脱磷效果显著优于 $2^{\#} \sim 4^{\#}$ 系统 $(P<0.05)$, 分别提高了 $13.53 \% 、 8.54 \%$ 和 $11.97 \%$ 。

在厌氧条件下, 兼性反硝化细菌能够利用硝态氮作为电子受体, 产生与氧同样的摄磷作用, 从而能将反

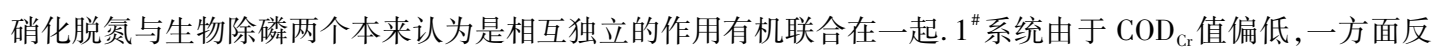
硝化菌无法获得充足的碳源进行反硝化, 另一方面聚磷菌也无法获得足够的碳源进行释磷, 因而氮和磷的 去除效果都不佳, 在系统中添加碳源后, 反硝化菌和聚磷菌都可获得相对较多的碳源进行反硝化和释磷, 所 以氮和磷的去除效率都随之提高 ${ }^{[22]} .2^{\#} \sim 4^{\#}$ 系统出水 $\mathrm{TN}$ 去除率分别比 $1^{\#}$ 系统提高了 $1.36 \% 、 2.40 \%$ 、 $2.93 \%$ 和 $3.80 \%$, 而 TP 去除率则提高了 $7.17 \% 、 12.16 \% 、 8.73 \%$ 和 $20.70 \%$, 说明聚磷菌在与反硝化菌对 碳源的竞争中占有优势. 因此, 添加碳源至人工湿地下层可以达到同步脱氮除磷的效果.

\section{3 结论}

1) 沿着水流方向 $\mathrm{COD}_{\mathrm{Cr}}$ 浓度逐渐降低, 随着 $\mathrm{COD}_{\mathrm{Gr}}$ 浓度的降低, 下层的硝态氮去除率较中层降低了 $1.63 \%, \mathrm{COD}_{\mathrm{Cr}}$ 浓度的降低导致微生物可利用的碳源缺乏, 从而阻碍了反硝化作用的进行, 因此碳源的添加 位置宜选择垂直流人工湿地中、下层.

2) 添加碳源后 $\mathrm{COD}_{\mathrm{Cr}}$ 出水浓度均低于进水, 说明千屈菜植物材料是人工湿地系统可供选择的有机碳源 材料, 不会引起二次污染. 不同位置添加固体碳源对 $\mathrm{COD}_{\mathrm{Cr}}$ 去除无影响. 
3) 补充千屈菜植物材料为反硝化提供了充足的电子供体, 提高了系统的脱氮效果, 碳源添加位置为湿 地下层时 TN 去除率最高. 表层系统氨氧化作用明显, 出水铵态氮浓度最高, 各系统对亚硝态氮和硝态氮去 除差异不明显,但对硝态氮都表现出良好的去除效果,下层系统硝化作用最完全.

4) 添加碳源后 TP 去除率得到提高, 不同添加位置对脱磷效果不造成显著影响. 底层系统脱磷效果最 佳. 聚磷菌在与反硝化菌对碳源的竞争中占有优势, 添加碳源至人工湿地下层可以同时达到脱氮除磷的 效果.

致谢: 张甬元老师在论文修改中给予了大量的指导和帮助, 刘保元、梁威等老师在实验设计和开展中给予了 指导和支持, 蔡林林、武俊梅、代娺然、鲁志营、张燕等同学在实验过程中提供帮助, 在此一并表示感谢.

\section{4 参考文献}

[ 1 ] 吴晓否. 人工湿地废水处理机理. 环境科学, 1994,16(3):83-86.

[ 2 ] 孙文杰,余宗莲,关艳艳等. 垂直流人工湿地净化污水的研究进展. 安全与环境工程, 2011, 18 (1):25-44.

[ 3 ] 赵联芳, 朱 伟, 赵建等. 人工湿地处理低碳氮比污染河水时的脱氮机理. 环境科学学报, 2006, 26 (11): 1821-1827.

[ 4 ] 王晓娟,张荣社. 人工湿地微生物硝化和反硝化强度对比研究. 环境科学学报,2006,26(2):225-229.

[ 5] 孙亚兵, 冯景伟, 田园春等. 自动增氧型潜流人工湿地处理农村生活污水的研究. 环境科学学报, 2006,26 (3): 404-408.

[ 6 ] 贺 锋, 吴振斌, 陶 菁等. 复合垂直流人工湿地污水处理系统硝化与反硝化作用. 环境科学, 2005, 26(1):47-50.

[ 7 ] 刘超翔,董春宏,李峰民等. 潜流式人工湿地污水处理系统硝化能力研究. 环境科学, 2003,24(1):80-83.

[ 8 ] 王 薇, 俞 燕, 王世和等. 人工湿地污水处理工艺与设计. 城市环境与城市生态, 2001, 14(1):59-62.

[ 9 ] International Water Association(IWA). Constructed wetland for pollution control process, performance, design and operation. London: IWA Publishing, 2000: 12-23.

[10] Kozub DD, Liehr SK. Assessing denitrification rate limiting factors in a constructed wetland receiving landfill leachate. Water Research, 1999, 40(3): 75-82.

[11] Fleming-Singer MS, Horne AJ. Enhanced nitrate removal efficiency in wetland microcosms using an episediment layer for denitrification. Environmental Science and Technology, 2002, 36(6):1230-1237.

[12] 陆松柳, 胡洪营. 人工湿地的反硝化能力研究. 中国给水排水, 2008, 24(7):63-69.

[13] Ingersoll TD, Baker LA. Nitrate removal in wetland microcosms. Water Research, 1998, 32(2):667-684.

[14] Huett DO, Morris SG, Smith G et al. Nitrogen and phosphorus removal from plant nursery runoff in vegetated and unvegetated subsurface flow wetlands. Water Research, 2005, 39:3259-3272.

[15] Lin YF, Jing SR, Wang TW et al. Effects of macrophytes and external carbon sources on nitrate removal from groundwater in constructed wetlands. Environmental Pollution, 2002, 119:413-420.

[16] 罗继红,王璐璐. 以农业废弃物作为反硝化脱氮固体碳源的研究现状. 现代农业科技, 2009, $7: 248-251$.

[17] Phipps RG, Crumpton WG. Factors affecting nitrogen loss in experimental wetlands with different hydrologic loads. Ecological Engineering, 1994, 3(4):399-408.

[18] 国家环境保护总局《水和废水监测分析方法》编委会. 水和废水监测分析方法: 第 4 版. 北京: 中国环境出版 社, 2002 .

[19] 余丽华,贺 锋,徐 栋等. 碳源调控下复合垂直流人工湿地脱氮研究. 环境科学, 2009,30(11): 3300-3305.

[20] Oh J, Silverstein J. Acetate limitation and nitrite accumulation during denitrification. Environmental Engineering, 1999, 125 (3) :234-242.

[21] 赵晨红,彭永臻,王淑莹等. 碳氮比对 UniFed SBR 工艺性能的影响. 环境科学, 2008,29(5):1210-1215.

[22] 贾文林, 吴 娟,武爱国等. 碳氮比对人工湿地污水处理效果的影响. 环境工程学报, 2010,4(4): 767-770. 